\title{
How Often Should Healthy Optic Nerves be Scanned for the Development of Glaucomatous Optic Neuropathy?
}

\author{
Paul Varner* \\ John J Pershing VAMC, USA \\ *Corresponding Author: Paul Varner, John J Pershing VAMC, USA.
}

Received: September 14, 2020

Published: September 23, 2020

(C) All rights are reserved by Paul Varner.

\begin{abstract}
Background: No data exist for the time period required for the conversion of healthy optic nerves to glaucomatous optic neuropathy (GON). It is not possible to predict which patients will develop glaucoma. Periodic screening of ONHs via nerve fiber layer (NFL) scans might allow for the early detection of GON.

Method: Retrospective chart review of patients who developed definitive unilateral $(\mathrm{n}=18)$ or bilateral $(\mathrm{n}=13)$ glaucoma to determine time course for conversion to/discovery of GON, as corroborated by optic nerve head (ONH) evaluation, NFL scans, and automated visual field (VF) perimetry.

Results: Median time of conversion for this cohort was 7 years for unilateral and 8 years for bilateral GON.

Conclusion: Screening ONHs/NFL at five-year intervals may be adequate for the detection of new cases of GON in its early stages.

Keywords: Glaucoma; Nerve Fiber Layer; Screening Frequency
\end{abstract}

\section{Introduction}

Glaucoma is now recognized as an optic neuropathy characterized by apoptosis of retinal ganglion cells (RGC) resulting in loss of the retinal nerve fiber layer (NFL, primarily composed of RGC axons), eventually yielding distinctive atrophic changes in the optic nerve head that result in corresponding visual field defects [1-3]. All of these components are part of and required for the diagnosis of glaucoma. This ophthalmic disease is incurable, irreversible and typically progressive, and the etiology for primary-open angle glaucoma (the most common form) remains unknown. Both congenital and adult-onset categories of glaucoma exist, with differentiation into various primary and secondary forms; however, all types result in the final common clinical pathway leading to glaucomatous optic neuropathy (GON).

Glaucoma is not a rare disease. The World Health Organization considers glaucoma to be the third most common source of vision impairment and blindness - trailing only refractive error and cataract in numbers [4] (both highly treatable). Estimates for global prevalence in populations aged 40 - 80 fall between $1-4 \%$, depending on type of glaucoma and ethnicity [5].

The costs of glaucoma to society are high. In 2004, an estimated $\$ 2.9$ billion was spent on direct costs of glaucoma in the US alone [6] - this does not include indirect expenditures and productivity losses. As financial burden increases with worsening disease severity [7], it becomes evident that earlier identification and intervention is advantageous to patients' quality of life as well as potentially reducing personal, family/caregiver and healthcare system economic burdens [8].

Unlike most ophthalmic diseases, glaucoma remains asymptomatic to patients until late in the course of the disease. The first visible indication of glaucoma for clinicians is suspicious optic nerve head (ONH) morphology - specifically, an excavating optic disk cupping [9] - interpreted clinically as enlargement of the cup-to-disk (c/d) ratio $\geq 0.7$ [10]. Vertical $c / d$ ratio has higher diagnostic value than horizontal $\mathrm{c} / \mathrm{d}$ ratio [11] and this correlates with the finding 
that superior and inferior NFL defects are more often associated with GON [12]. In comparing eyes, a difference in $\mathrm{c} / \mathrm{d}$ ratios of 0.2 or more is present only $4 \%$ of the time [13] - with outliers serving as important markers for the identification of unilateral glaucoma. Unfortunately, evaluation of $\mathrm{c} / \mathrm{d}$ ratios is subjective and occurs with variable intra- and inter-observer reproducibility with respect to ONH baseline characteristics, identification of glaucoma suspects, and in the detection of disease progression [14-18].

Fortunately, objective ONH and NFL evaluations with progression analysis are now possible via optical coherence tomography (OCT). This is critically important in the detection of glaucoma considering that RGC losses as determined by NFL thickness measurements via OCT are highly correlated with glaucoma [19]. NFL defects outside statistical norms are recognized to be important in the diagnosis of early glaucoma [20] and precede visual field (VF) defects as evidenced by both NFL photography [21] and OCT data (Domanico I., et al. IOVS 2011;52:ARVO E-Abstract 5077). In fact following the pathophysiological processes involved - NFL losses are required prior to the development of VF losses from glaucoma [22] and structural (NFL) defects must match functional (VF) losses for definitive diagnosis. Thus, NFL and/or VF defects discovered when $\mathrm{ONH}$ appearance indicates a c/d ratio $<0.7$ with normal NFL scans logically preclude classification as GON.

Although some NFL loss is expected with increasing age [23] the appearance of the ONH changes very little over time, with normal senescent changes (specifically, NFL loss through RGC death) possibly accounting for only a 0.1 increase in c/d ratio between ages 30 and 70 [24] - an amount insufficient to be mistaken for glaucomatous progression [25].

For primary open-angle glaucoma (POAG) there is a clear association with age - a consistent finding across many populationbased studies among various ethnic groups [26,27] - and this form is rare in persons under the age of 40 . Other forms of glaucoma (again, defined through ONH damage + NFL/VF loss) vary somewhat from POAG in their demographics. The average age for development of angle-closure glaucoma is 60 [28]. Of the most common. secondary forms of open-angle glaucoma, pigmentary glaucoma is more often found in the 40s $[29,30]$ median age for the diagnosis of traumatic glaucoma is 45 (although males 20 - 29 are in highest risk group) [31] pseudoexfoliation glaucoma is more common for patients in their 60s and 70s [32,33] and the development of uveitic glaucoma may have no age predilection [34] presumably related to the underlying type of uveitis.

It should be emphasized, however, that for angle-closure and secondary open-angle forms of glaucoma, the associated findings - and patient symptoms in some cases - involve different risk stratification regarding development of secondary GON although diligent practitioners are unlikely to miss these factors.

Because there is currently no way to predict which patients will ultimately develop GON, the clinical challenge is in the identification of new cases of glaucoma at the earliest stage possible. Eye providers need an objective way to screen individuals who were previously normal in order to provide a preliminary identification for a possible eye disease that is expensive, potentially visually significant, yet asymptomatic early in its course. Preferably, such a monitoring program would be an ongoing process throughout the periods of greatest incidence of glaucoma development.

The characteristics of an ideal screening program have been studied over the past 50 years (Table 1) [35,36]. ONH screening for the detection of asymptomatic glaucoma appears to meet these criteria. Despite limitations (patient cooperation during testing, degraded scan quality related to poor ocular surface or pupillary size, paradoxical results etc.) NFL scans via OCT have appropriate sensitivity, specificity and positive predictive value in the detection of GON [37] - especially for early disease [38,39] - and are reproducible [40]. As a final note, it is crucial to remember that NFL testing in and of itself is not a diagnostic test for GON; rather, it is part of a compendium of tests required to make the definitive diagnosis.

- The condition sought should be an important health problem.

- The epidemiology and natural history of the condition, including development from latent to declared disease, should be adequately understood.

- There should be a recognizable latent or early symptomatic stage.

- There should be a suitable test or examination.

- The test should be acceptable to the population - clinically, socially, ethically.

- There should be an agreed policy on whom to treat as patients.

- $\quad$ There should be an accepted treatment for patients with recognized disease and coordinated follow-up with an expected benefit.

- Facilities for diagnosis and treatment should be available.

- The cost of case-finding (including diagnosis and treatment of patients diagnosed) should be economically balanced in relation to possible expenditure on medical care as a whole.

- Case-finding should be a continuing process and not a "once and for all" project.

Table: Characteristics of a screening test [35]. 
Clearly there is a clinical progression from a healthy to a damaged $\mathrm{ONH}$ in glaucoma and it is equally apparent that not all individuals will go on to develop GON. To date there is no intraocular pressure-independent data available regarding the conversion of healthy ONHs to glaucoma. An indirect estimate for the time course of conversion can be made based on what is currently known about disease progression in patients known to have the disease.

One of the most important factors involved in glaucoma management is the concept of rate of progression [41]. Although various forms of glaucoma can progress to marked visual disability, clinicians must bear in mind that this is not the most common outcome, and that POAG is not expected to result in bilateral blindness - even 13 - 16 years after the first definite VF losses [42]. The rate of progression of glaucomatous disease is typically assessed by VF decline - the surrogate marker for optic neuropathy. Consensus criteria for VF loss do not exist; however, it is instructive to note that two large clinical trials of VF progression in glaucoma (EMGT, CIGTS) reported VF progression over 6 - 8 years and not shorter periods of time $[43,44]$.

VF deterioration for other glaucoma subtypes is limited (many reports remain biased by intraocular pressure criteria and not VF defects to make diagnosis); however, progression for angle-closure, pigmentary and exfoliative forms has been reported to take place over 6+ years [45] and 7 - 9 years for uveitic glaucoma [46,47]. In the angle-recession form of traumatic glaucoma VF losses were reported over a 4-year period of follow-up [48].

Data on conversion from ocular hypertension is conflicting between the Ocular Hypertension Treatment Study [49] and the European Glaucoma Prevention Study [50]; however, in the present context, it is important to notice that both studies were carried out over 5-year periods of time and not shorter follow-up intervals.

Considering that VF progression is generally slow after a definitive diagnosis of POAG, what can be said about screening frequency for patients with normal ONHs - only a small fraction of which will go onto develop GON?

In practice, subjects at higher risk for the development of GON are often labelled glaucoma suspects, and then followed at shorter intervals between clinical re-examinations in order to determine whether conversion to clinically evident glaucoma has occurred. A glaucoma suspect has been defined as "an individual with clini- cal findings and/or a constellation of risk factors that indicate an increased likelihood of developing primary open-angle glaucoma" [51]. However, this designation remains controversial [52] and there are no rigorous studies delineating specific clinical characteristics to unequivocally distinguish this taxonomy. To further confound this issue, there is no ICD-10 designator for glaucoma suspect based on optic nerve morphology. Characterizing a patient as having "preglaucoma" is even less useful clinically, and a throwback to the days of intraocular pressure dogma [53].

A more accurate designator would be glaucoma suspect by ONHs or by ONHs/NFL defects, but this is only a temporary label until consistent NFL and VF testing can be obtained. In the event VFs are full or repeatably variable, other sources of optic neuropathy should be explored. Normal NFL scans effectively rule out glaucoma [54] indicating that the appearance of enlarged c/d ratios are a normal variation, or "physiological cupping of ONHs." Although it has been suggested that there is no need to obtain NFL scans more often than once per year [54] even this interim may be too frequent.

Given that the time course for the development of NFL losses leading to GON and accompanying VF losses for the most common form of asymptomatic glaucoma occurs over the course of several years; that it is not currently possible to predict which patients will ultimately develop glaucoma of any form; and that NFL scans are now the most sensitive and specific way to detect early GON, at what intervals could ONHs be screened via NFL evaluation in order to detect glaucoma? There is no prospective data available to answer this important clinical question.

\section{Purpose of the Study}

The purpose of this retrospective case series is to provide a preliminary estimate to answer this question in order to set the stage for identifying a screening time frame for the purpose of detecting GON in its earliest phase.

\section{Methods}

All patients evaluated and other charts administratively reviewed by the author during a typical 6-month window (September 2019 through February 2020) were evaluated to determine the presence of a diagnosis of any form of glaucoma and whether progression could be determined - or discovered in the case of patients who had been previously diagnosed and then lost to followup. ONH photos, Cirrus HD-OCT NFL scans, and Humphrey VF data 
were reviewed for completion and cases with incomplete records or paradoxical results precluding decisive glaucoma diagnosis were excluded from review.

Included patients had GON with c/d ratio $\geq 0.7$, marked repeatable NFL defects in one or both vertical quadrants, and corresponding repeatable characteristic VF defects in one or both eyes. The algorithm for identification of GON is provided in figure 1 and a representative case with correlated clinical findings is presented in figure 2. Ages at the time of conversion/discovery, gender, type of glaucoma and eye(s) of regard were noted. For each of these cases, the elapsed time between non-glaucomatous $\mathrm{ONH}$ and conversion to/discovery of GON was determined via the change between full VFs and the development of repeatable scotomata consistent with ONH appearance and corresponding NFL losses.

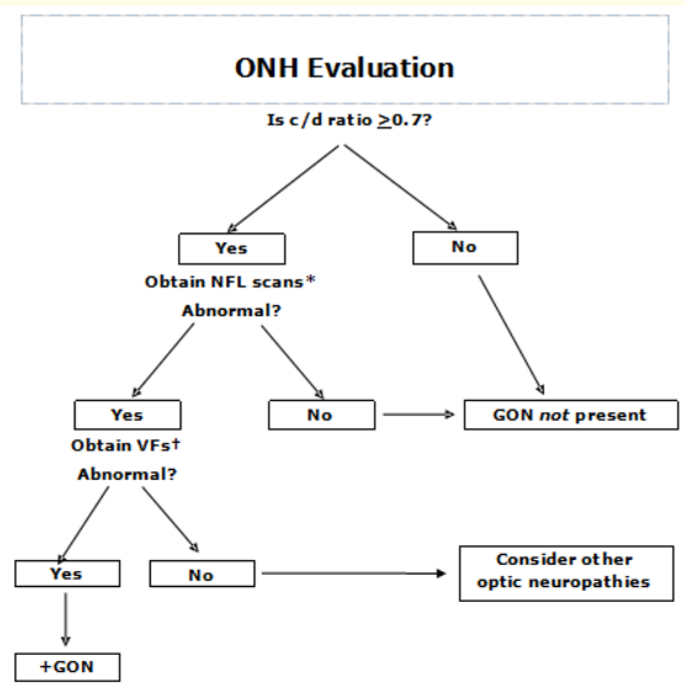

Figure 1: Algorithm for the determination of glaucomatous optic neuropathy.

*: If reliable and consistent with ONH appearance

$\dagger$ : If reliable, with repeatable defects, and consistent with $\mathrm{ONH}$ appearance and NFL scans.

Abbreviations: GON: Glaucomatous Optic Neuropath; NFL: Nerve Fiber Layer; ONH: Optic Nerve Head; VF: Visual Field.
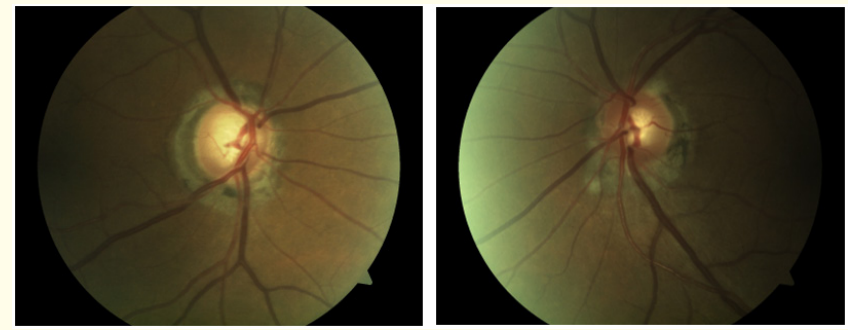

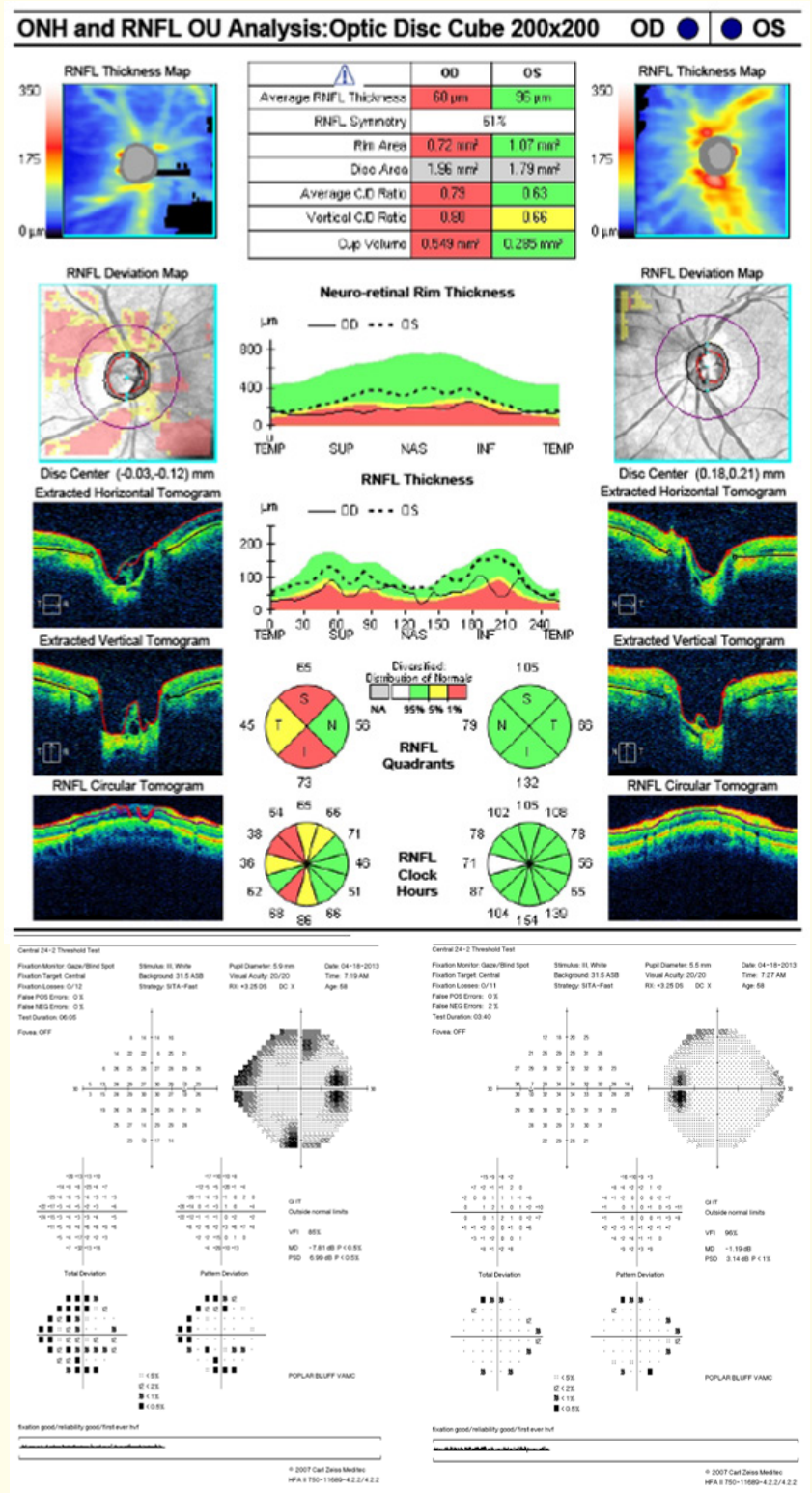

Figure 2: Traumatic glaucoma OD in 65 year-old male. Within limits of patient testing reliability, visual field scotomata match nerve fiber layer defects and optic nerve head appearance.

As all clinicians are well-aware, there continue to be paradoxical results that defy a conclusive diagnosis of glaucoma. ONHs found with $0.5 \mathrm{c} / \mathrm{d}$ ratios can have unusual objective NFL defects, confounded by subjective non-corresponding VF defects (informally called "red disease" based on color-coding of NFL scans, Figure 3). Likewise, $0.8 \mathrm{c} / \mathrm{d}$ ratios with entirely normal NFL scans create another source of confusion (colloquially referred to as "green disease", Figure 4). These ambiguous cases were excluded from this review, as these findings may reflect various artifacts, or - if veritable - congenital defects or other subtle optic neuropathies. 

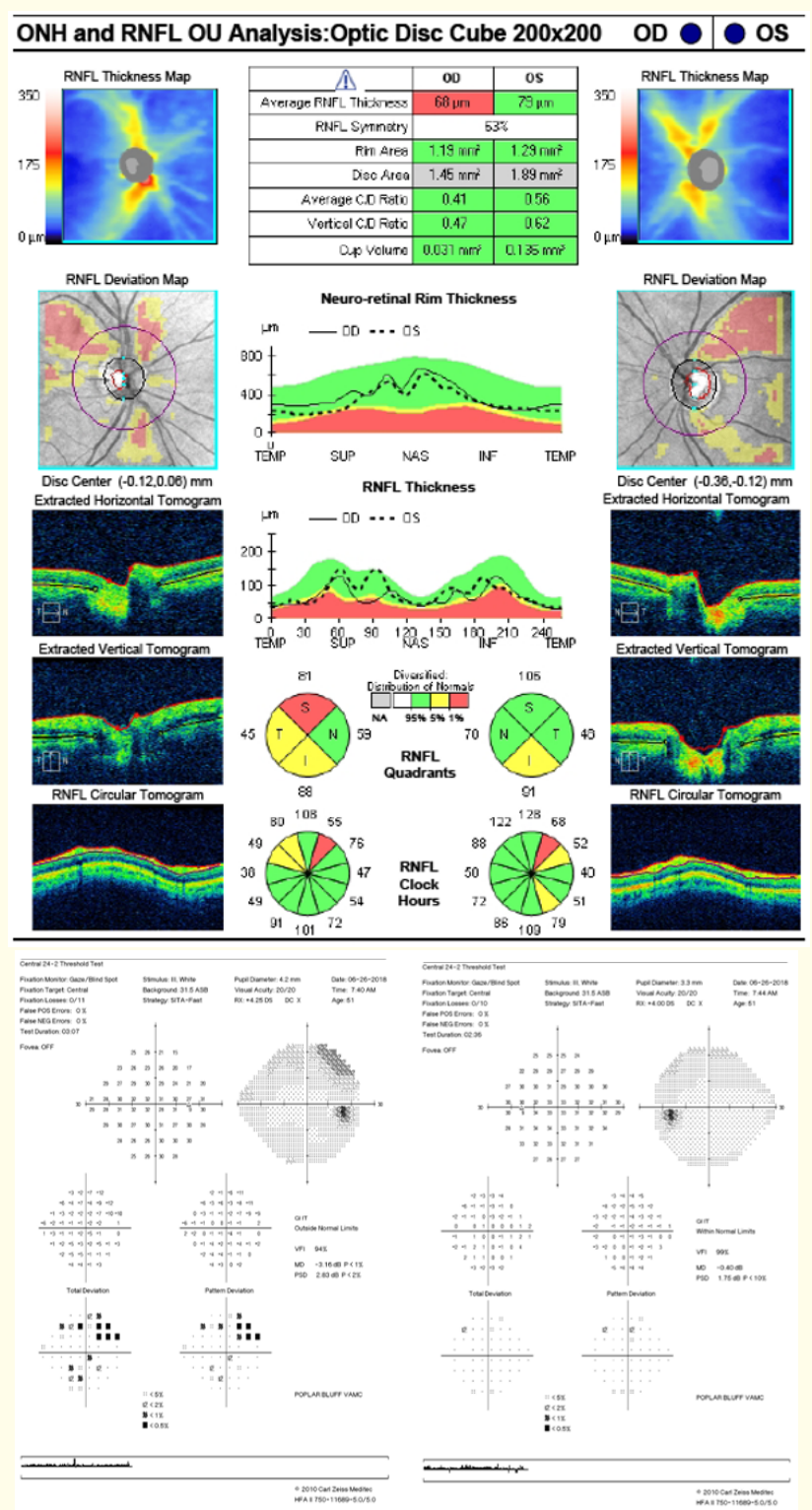

Figure 3: "Red Disease" in a 53 year-old male. Note non-correspondence between NFL scans and HVFs.

\section{Results}

For the period of subject identification involved, complete information and unambiguous time frames for conversion/discovery were found for 18 unilateral and 13 bilateral subjects with glaucoma. Only one female patient was identified in each group. Of the unilateral cases nine cases were found to be traumatic in origin, seven were unspecified open-angle glaucoma, and there was one each of pigmentary, and mixed-mechanism (trauma/ACG) glauco- ma. The average age for these patients was 68 years with a range of 48 - 85, and the median time between conversion/discovery was seven years (vs a mean of nine years) with a range of 2 - 23 years. With respect to conversion/discovery times, median values were less likely to be skewed by outliers for this small sample size.

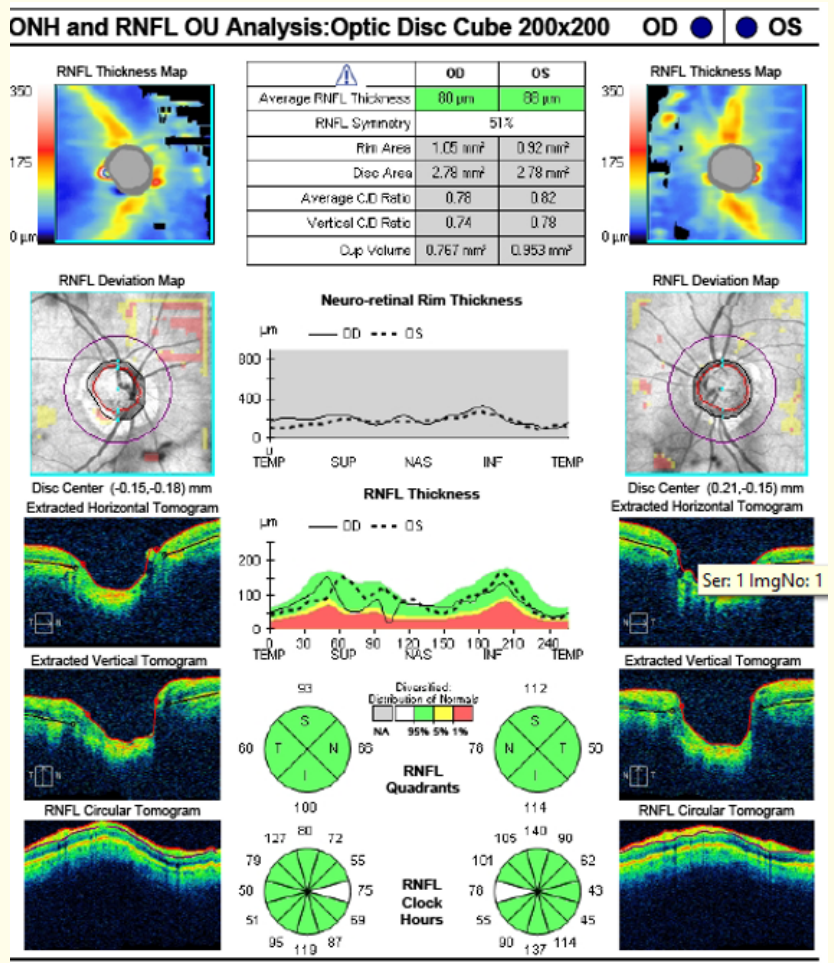

Figure 4: "Green Disease" in an 81 year-old male. ONH evaluation would seem to predict NFL defects, yet NFLs remain intact.

Of special note are the seven cases of unspecified open-angle glaucoma. It seems counterintuitive that there is such a clinical entity as "unilateral POAG" as this idiopathic pathophysiology appears to affect both eyes most of the time. In each of these cases, every effort has been made to review history for ophthalmic surgical history, ocular trauma, and even consistent head positioning during sleep, as well as to identify clinical findings associated with angle closure and/or secondary forms of glaucoma (pigment dispersion, pseudoexfoliation, subtle signs of prior uveitis, etc). However, for these seven cases, there were no further clues to the source of unilaterality. This perplexing clinical situation is not novel $[55,56]$ and remains inexplicable.

Ten of the bilateral cases were POAG, two were mixed mechanism (phacomorphic/POAG vs POAG and traumatic/POAG vs 
POAG), and one was pigmentary glaucoma. The average age for these cases was also 68 years with a range of 41 to 91 and the median time between conversion/discovery was eight years (vs a mean of ten years) with a range of 3 - 28 years.

\section{Discussion and Conclusion}

Retrospective anecdotal findings for this small group of cases with known glaucoma give credence to the clinical intuition that the conversion from healthy optic nerves to GON is slow and occurs over a period of several years. A frequency screening threshold set at five years would have picked up 15/18 (83\%) of these unilateral cases and $10 / 13(80 \%)$ of patients $(20 / 26$ of eyes) in the bilateral group. Of the six patients that converted or were discovered in time periods $<5$ years, only one of the unilateral patients was found to have $<20 / 20$ vision when detected. In other words, GON was not the reason these patients were returning for follow-up ophthalmic care.

It may be quickly deduced that these results have a very clear application within the field of telemedicine. As teleretinal screening programs expand beyond searching for diabetic retinopathy, it seems that the addition of NFL scanning might be appropriate for selected ONH presentations. The integration of ONH photos/NFL scans via remote technology could be used to improve early detection of converters and to prevent unnecessary face-to-face screening appts that might require long travel distances for patients and create additional burdens on existing health care resources.

As with all screening programs, such an intervention is a matter of providing the right test for the right patients at the right place and at the right time. As a direct application, asymptomatic patients not receiving any eye care could be screened with standard retina/ ONH photo sets within the primary health care setting, starting at age 40. For negative initial screenings, then - in the absence of any visual, ocular discomfort or other ophthalmic symptoms - the imaging protocol could be repeated at five-year intervals.

For those patients found to have $\mathrm{c} / \mathrm{d}$ ratios $\geq 0.7$, additional NFL scans could be obtained remotely. Positive screenings based on pre-set $\mathrm{ONH} / \mathrm{NFL}$ criteria would be followed up with face-to-face eye examination, presumably including automated perimetry, with subsequent follow-up determined by the amalgam of those findings. Special attention would be required to identify false positives and to uncover missed, false negatives to refine the procedure. $\mathrm{Ob}$ viously, society and payors must balance cost of screening versus yield for frequency.
These preliminary, anecdotal findings must be confirmed with solid prospective data; however, these preliminary findings suggest every 5 years would be adequate in detecting new cases of GON in the early stages.

\section{Disclosures}

The views expressed in this article are those of the author and do not necessarily represent the position of the Department of Veterans Affairs.

The author reports no proprietary or commercial conflicts of interest for any product mentioned or concept discussed in this article.

\section{Acknowledgements}

The author would like to thank Antonia Varner for the graphic designs.

\section{Bibliography}

1. McKinnon SJ. "Glaucoma, apoptosis, and neuroprotection". Current Opinion in Ophthalmology 8 (1997): 28-37.

2. Fortune B., et al. "Relating Retinal Ganglion Cell Function and Retinal Nerve Fiber Layer (RNFL) Retardance to Progressive Loss of RNFL Thickness and Optic Nerve Axons in Experimental Glaucoma". Investigative Ophthalmology and Visual Science 56 (2015): 3936-3944.

3. Chen CL., et al. "Peripapillary Retinal Nerve Fiber Layer Vascular Microcirculation in Eyes With Glaucoma and SingleHemifield Visual Field Loss". JAMA Ophthalmology 135 (2017): 461-468.

4. World Health Organization. "Blindness and Vision Impairment" (2019).

5. Tham Y-C., et al. "Global prevalence of glaucoma and projections of glaucoma burden through 2040: a systematic review and meta-analysis". Ophthalmology 121 (2014): 2081-2090.

6. Rein DB., et al. "The economic burden of major adult visual disorders in the United States". Archives of Ophthalmology 124 (2006): 1754-1760.

7. Traverso CE., et al. "Direct costs of glaucoma and severity of the disease: a multinational long term study of resource utilisation in Europe". British Journal of Ophthalmology 89 (2005): 1245-1249. 
8. Varma, R., et al. "An Assessment of the Health and Economic Burdens of Glaucoma". American Journal of Ophthalmology 152 (2011): 515-522.

9. Tanito M., et al. "Differentiation of glaucomatous optic discs with different appearances using optic disc topography parameters: The Glaucoma Stereo Analysis Study". PLoS ONE 12 (2017): e0169858.

10. Quigley HA. "New paradigms in the mechanisms and management of glaucoma". Eye 19 (2005): 1241-1248.

11. Jonas JB., et al. "Ranking of optic disc variables for detection of glaucomatous optic nerve damage". Investigative Ophthalmology and Visual Science 41 (2000): 1764-1773.

12. Jonas JB and Schiro D. "Localised wedge shaped defects of the retinal nerve fibre layer in glaucoma". British Journal of Ophthalmology 78 (1994): 285-290.

13. Jonas JB., et al. "Optic disc, cup and neuroretinal rim size, configuration and correlations in normal eyes". Investigative Ophthalmology and Visual Science 29 (1988): 1151-1158.

14. Tielsch JM., et al. "Intraobserver and interobserver agreement in measurement of optic disc characteristics". Ophthalmology 95 (1988): 350-356.

15. Sturmer J., et al. "Intra- and inter-observer variation of optic nerve head measurements in glaucoma suspects using discdata". International Ophthalmology 16 (1992): 227-233.

16. Coleman AL., et al. "Interobserver and intraobserver variability in the detection of glaucomatous progression of the optic disc". Journal of Glaucoma 5 (1996): 384-389.

17. Azuara-Blanco A., et al. "Detection of changes of the optic disc in glaucomatous eyes: clinical examination and image analysis with the Topcon Imagenet system". Acta Ophthalmologica Scandinavica 78 (2000): 647-650.

18. Harper R., et al. "Observer variability in optic disc assessment: implications for glaucoma shared care". Ophthalmic and Physiological Optics 20 (2000): 265-273.

19. Guedes V., et al. "Optical coherence tomography measurement of macular and nerve fiber layer thickness in normal and glaucomatous human eyes". Ophthalmology 110 (2003): 177-189.

20. Jonas JB and Schiro D. "Localised wedge shaped defects of the retinal nerve fibre layer in glaucoma”. British Journal of Ophthalmology 78 (1994): 285-290.
21. Sommer A., et al. "Clinically detectable nerve fiber atrophy precedes the onset of glaucomatous field loss". Archives of Ophthalmology 109 (1991): 77-83.

22. Alasil T., et al. "Correlation of retinal nerve fiber layer thickness and visual fields in glaucoma: a broken stick model". American Journal of Ophthalmology 157 (2014): 953-959.

23. Leung CK., et al. "Retinal nerve fiber layer imaging with spectral-domain optical coherence tomography: a prospective analysis of age-related loss". Ophthalmology 119 (2012): 731737.

24. Garway-Heath DF., et al. "Aging changes of the optic nerve head in relation to open angle glaucoma". British Journal of Ophthalmology 81 (1997): 840-845.

25. Moya FJ., et al. "Effect of aging on optic nerve appearance: a longitudinal study". British Journal of Ophthalmology 83 (1999): 567-572.

26. Tuck MW and Crick RP. "The age distribution of primary open angle glaucoma”. Ophthalmic Epidemiology 5 (1998): 173-183.

27. American Academy of Ophthalmology. Primary Open-Angle Glaucoma: Epidemiology (2013).

28. Khazaeni B and Khazaeni L. "Acute Closed Angle Glaucoma". Treasure Island (FL): Stat Pearls Publishing. E-Book (2020).

29. Scheie HG and Cameron JD. "Pigment dispersion syndrome: a clinical study". British Journal of Ophthalmology 65 (1981): 264-269.

30. Siddiqui Y., et al. "What is the risk of developing pigmentary glaucoma from pigment dispersion syndrome?" American Journal of Ophthalmology 135 (2003): 794-799.

31. Ajita KO., et al. "Survey of Traumatic Glaucoma in a Tertiary Hospital". Journal of Trauma and Treatment 6 (2017): 1.

32. Karger RA., et al. "Estimated incidence of pseudoexfoliation syndrome and pseudoexfoliation glaucoma in Olmsted County, Minnesota". Journal of Glaucoma 12 (2003): 193-197.

33. Plateroti P., et al. "Pseudoexfoliation Syndrome and Pseudoexfoliation Glaucoma: A Review of the Literature with Updates on Surgical Management". Journal of Ophthalmology (2015): 370371.

34. Siddique SS., et al. "Glaucoma and uveitis". Survey of Ophthalmology 58 (2013): 1-10. 
35. Wilson JMG and Jungner G. "Principles and practice of screening for disease. Geneva: World Health Organization; Public Health Papers 34 (1968).

36. Dobrow MJ., et al. "Consolidated principles for screening based on a systematic review and consensus process". Canadian Medical Association Journal 190 (2018): E422-E429.

37. Gondal TM., et al. "Accuracy of the retinal nerve fiber layer measurements by Stratus optical coherence tomography for perimetric glaucoma". Journal of the College of Physicians and Surgeons Pakistan 21 (2011): 749-752.

38. Wu H., et al. "Diagnostic capability of spectral-domain optical coherence tomography for glaucoma". American Journal of Ophthalmology 153 (2012): 815-826.

39. Zhang X., et al. "Comparison of Glaucoma Progression Detection by Optical Coherence Tomography and Visual Field". American Journal of Ophthalmology 184 (2017): 63-74.

40. Wu H., et al. "Reproducibility of retinal nerve fiber layer thickness measurements using spectral domain optical coherence tomography". Journal of Glaucoma 20 (2011): 470-476.

41. Heijl A., et al. "Rates of visual field progression in clinical glaucoma care". Acta Ophthalmologica 91 (2013): 406-412.

42. Quigley HA., et al. "Rate of progression in open-angle glaucoma estimated from cross-sectional prevalence of visual field damage". American Journal of Ophthalmology 122 (1996): 355-363.

43. Leske MC., et al. "Factors for glaucoma progression and the effect of treatment: the early manifest glaucoma trial". Archives of Ophthalmology 121 (2003): 48-56.

44. Musch DC., et al. "Visual field progression in the Collaborative Initial Glaucoma Treatment Study: the impact of treatment and other baseline factors". Ophthalmology 116 (2009): 200-207.

45. De Moraes CG., et al. "Visual field progression outcomes in glaucoma subtypes”. Acta Ophthalmologica 91 (2013): 288293.

46. Din NM., et al. "Difference in glaucoma progression between the first and second eye after consecutive bilateral glaucoma surgery in patients with bilateral uveitic glaucoma". Graefe's Archive for Clinical and Experimental Ophthalmology 254 (2016): 2439-2448.

47. Sharon Y., et al. "Uveitic glaucoma: long-term clinical outcome and risk factors for progression". Ocular Immunology and Inflammation 25 (2017): 740-747.
48. Ng DS., et al. "Angle-recession glaucoma: long-term clinical outcomes over a 10-year period in traumatic microhyphema". International Ophthalmology 35 (2015): 107-113.

49. Kass MA., et al. "The Ocular Hypertension Treatment Study: a randomized trial determines that topical ocular hypotensive medication delays or prevents the onset of primary openangle glaucoma”. Archives of Ophthalmology 120 (2002): 701713.

50. European Glaucoma Prevention Study (EGPS) Group. "Results of the European Glaucoma Prevention Study". Ophthalmology 112 (2005): 366-375.

51. Prum BE., et al. "Primary Open-Angle Glaucoma Suspect Preferred Practice Pattern(®) Guidelines". Ophthalmology 123 (2016): 112-151.

52. Ahmad SS. "Glaucoma suspects: a practical approach". Taiwan Journal of Ophthalmology 8 (2018): 74-81.

53. Gradle HS. "Preglaucoma”. American Journal of Ophthalmology 29 (1946): 520-523.

54. Chang RT and Singh K. "Glaucoma suspect: diagnosis and management”. The Asia-Pacific Journal of Ophthalmology 5 (2016): 32-37.

55. Etienne R. "Unilateral glaucoma”. British Journal of Ophthalmology 56 (1972): 254-258.

56. Sangawe JL. "Unilateral Glaucoma”. Tropical and Geographical Medicine 38 (1986): 70-72.

\section{Assets from publication with us}

- Prompt Acknowledgement after receiving the article

- Thorough Double blinded peer review

- Rapid Publication

- Issue of Publication Certificate

- High visibility of your Published work

Website: www.actascientific.com/

Submit Article: www.actascientific.com/submission.php

Email us: editor@actascientific.com

Contact us: +919182824667 